\title{
Single and Coupled Metasurfaces For Tunable Polarization-Sensitive Terahertz Filters
}

\author{
Andriy E. Serebryannikov \\ Adam Mickiewicz University \\ Faculty of Physics \\ 61-614 Poznan, Poland \\ email: andser@amu.edu.pl
}

\author{
Akhlesh Lakhtakia \\ Pennsylvania State University \\ Department of Engineering Science \\ and Mechanics, University Park, PA \\ 16802, USA
}

\author{
Ekmel Ozbay \\ Nanotechnology Research Center - \\ NANOTAM, Bilkent University \\ 06800 Ankara, Turkey \\ email: ozbay@bilkent.edu.tr
}

\begin{abstract}
We simulated the transmission of terahertz waves through a single metasurface and two coupled metasurfaces that comprise H-shaped subwavelength resonators made of InAs, a magnetically tunable material. The magnetostatic field was varied from 0 to $1 \mathrm{~T}$. The obtained results demonstrate that the substrate permittivity and the coupling of metasurfaces can significantly affect filtering performance as well as the possibility of tuning for different orientations of the magnetostatic field.
\end{abstract}

Keywords—subwavelength resonator; stop band; metasurface

\section{INTRODUCTION}

Metasurfaces that are arrays of subwavelength resonating elements have been the focus of research for more than a decade [1]. For the terahertz regime, they can be fabricated by using vapor deposition, lithography, and etching [2]. The typical metasurface comprises metallic resonating elements printed on a dielectric substrate. An emerging alternative is to use non-metallic resonators [3]. This alternative is especially promising for metasurfaces that can be dynamically tunable via electrical, magnetic, thermal, or other mechanisms [4].

We simulated the transmission of linearly polarized plane waves through metasurfaces that are periodic arrays of $\mathrm{H}$ shaped resonators made of InAs. This material is well suitable for tuning in the terahertz regime $(0.5$ to $5.5 \mathrm{THz})$, as its relative permittivity tensor is strongly sensitive to the strength of the applied magnetostatic field $\left(\mathrm{B}_{0}\right)$. Thus, it is expected that even small amounts of InAs like those required for subwavelength resonators can be sufficient to obtain strong sensitivity of the entire metasurface to variations of the magnetostatic field.

Simulations were performed by using the frequency domain solver of CST Microwave Studio (www.cst.com), a commercial software. Full details of a large part of the results of our investigations are available elsewhere [5]. Here, we briefly present results for a single metasurface and two identical coupled metasurfaces, for two different dielectric substrates, when $\mathrm{B}_{0}=0$ and $\mathrm{B}_{0}>0$. Our focus is on the effects on tunability by the substrate's relative permittivity scalar, the

This work was supported by National Centre of Science, Poland under the project MetaSel DEC-2015/17/B/ST3/00118; by TUBITAK under project nos. 113E331, 109A015, and 109E301; and under projects DPT-HAMIT, DPT-FOTON, and NATO-SET-193; AL thanks the Charles Godfrey Binder Endowment at Penn State; EO thanks the Turkish Academy of Sciences. choice of magnetostatic field orientation (either Faraday or Voigt), and the coupling between two metasurfaces.

\section{GeOMetry, Results AND Discussion}

\section{A. Geometry}

The unit cells of a single metasurface $(N=1)$ and two coupled metasurfaces $(N=2)$ are depicted in Fig. 1. The array period is denoted by $a$. Each $\mathrm{H}$-shaped resonator made of InAs consists of two $w \times h$ sections and one $w \times l$ section of thickness $t$, see Figs. 1(a,b). The substrate thickness is denoted by $b$. The case of two coupled metasurfaces is obtained just by placing two identical arrays of resonating elements on the opposite faces of the dielectric substrate, as shown in Fig. 1(c). Calculations were performed for $a=15.56 \mu \mathrm{m}, h=14 \mu \mathrm{m}, l=9 \mu \mathrm{m}, w=2.5 \mu \mathrm{m}, t=0.5 \mu \mathrm{m}$, and $b=2.5 \mu \mathrm{m}$, while substrate's relative permittivity scalar is either $\varepsilon_{d}=2.1$ or $\varepsilon_{d}=5.8$.

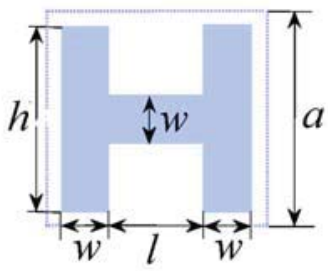

(a)

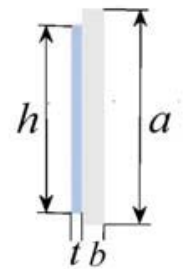

(b)

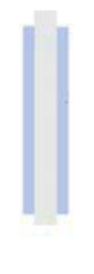

(c)
Fig. 1. Schematics of unit cells: (a) front view, and side views of (b) a single metasurface and (c) two identical coupled metasurfaces. The resonator and the substrate are shown in blue and grey, respectively.

InAs is a gyroelectric material with the relative permittivity tensor controllable by the external magnetostatic field $[3,5]$. When $\mathrm{B}_{0}=0$, InAs functions like an isotropic Drude metal. Anisotropy comes to the play when $\mathrm{B}_{0}>0$ : the diagonal elements of the relative permittivity tensor change their values from large positive to small, and the non-diagonal ents from large positive to zero value, depending on the frequency and value of $\mathrm{B}_{0}$.

The $x, y$, and $z$ axes are directed along the horizontal resonator arm, the vertical resonator arms, and perpendicularly to the resonator plane, respectively. The magnetostatic field 


\section{NUSOD 2016}

may be oriented along the $z$ axis (Faraday configuration), the $x$ axis (Voigt-X configuration), or the $y$ axis (Voigt-Y configuration). The structures are illuminated by a plane wave propagating along the $z$ axis, the plane wave being either $x$ polarized ( $\mathbf{E} \| \mathrm{x}$ axis) or $y$ polarized ( $\mathbf{E} \| \mathrm{y}$ axis).

\section{B. Transmission Coefficients}

Figure 2 shows the transmission coefficients for a single metasurface and two coupled metasurfaces when $\varepsilon_{d}=2.1$, for four combinations of the strength and orientation of magnetostatic field. Bandstop filtering is achieved for all cases considered.
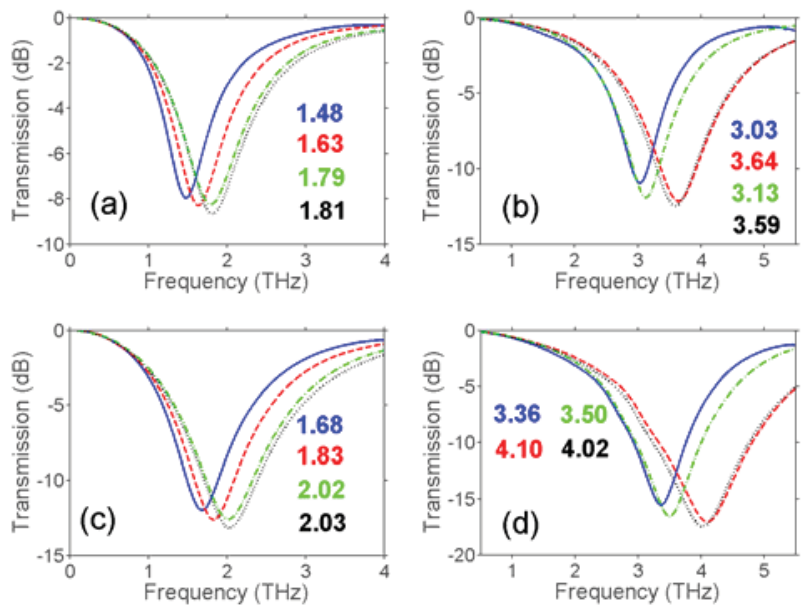

Fig. 2. Spectrums of the co-polarized transmission coefficients (a,c) $\left|\tau_{x x}\right|$ and (b,d) $\left|\tau_{y y}\right|$ for (a,b) $N=1$ and (c,d) $N=2$, for Faraday (solid blue line), Voigt-Y (dashed red line), Voigt-X (dash-dotted green line) configurations for $\mathrm{B}_{0}=1 \mathrm{~T}$, and for $\mathrm{B}_{0}=0$ (dotted black line); $\varepsilon_{d}=2.1$.

Colored numbers indicate center frequencies of the stopbands in $\mathrm{THz}$.

The following conclusions were drawn from Fig. 2. The center frequency of the stopband strongly depends on the polarization state of the incident plane wave. The effect of magnetostatic field is strong for both polarization states of the incident plane wave for the Faraday configuration, intermediate for the $x$-polarization state but vanishing for the $y$-polarization state for the Voigt-Y configuration, and vanishing for the $x$-polarization state but intermediate for the $y$-polarization state for the Voigt-X configuration. Application of the magnetostatic field generally redshifts the center frequency of the stop band. Addition of the second metasurface blueshifts the center frequency due to capacitive coupling, and deepens the transmission minimums. These conclusions are quite general and thus provide huge freedom for design.

For $N=1$, the stopband is tunable for the Faraday configuration in Figs. 2(a) and 2(b) over $320 \mathrm{GHz}$ and 560 $\mathrm{GHz}$, respectively; over $180 \mathrm{GHz}$ for the Voigt-Y configuration in Fig. 2(a); and over $470 \mathrm{GHz}$ for the Voigt-X configuration in Fig. 2(b). Likewise for $N=2$, the stop band is tunable for the Faraday configuration in Figs. 2(c) and 2(d) over $350 \mathrm{GHz}$ and $670 \mathrm{GHz}$, respectively; over $200 \mathrm{GHz}$ for the Voigt-Y configuration in Fig. 2(c); and over $530 \mathrm{GHz}$ for the Voigt-X configuration in Fig. 2(d). Hence, one can obtain better performance by coupling two metasurfaces. For $0<\mathrm{B}_{0}$ $<1 \mathrm{~T}$, an almost monotonous modification of the spectrum takes place that allows one to perform a fine tuning in addition to a sharp switching. The thickness-to-wavelength ratio $(2 t+b) / \lambda_{0}<6.5 \times 10^{-2}$ in the considered frequency range even for $N=2$; thus, the filters are electrically thin.

One more powerful tool of tailoring transmission and filtering characteristics is connected with the substrate's relative permittivity scalar. Figure 3 presents the same data as in Fig. 2 except that $\varepsilon_{d}=5.8$. One can see that all the features observed in Fig. 2 redshift. This effect is quite expected but its quantification needs further research.
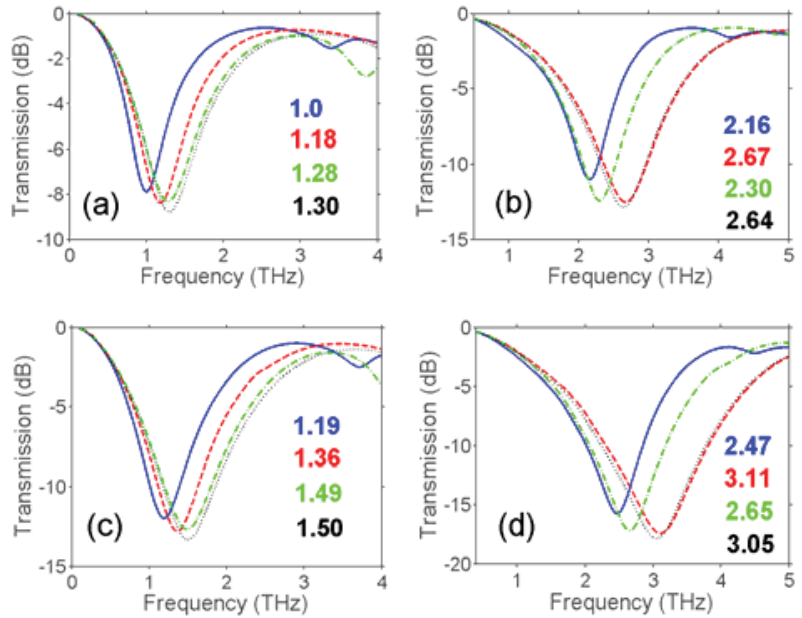

Fig. 3. Same as Fig. 2 but for $\varepsilon_{d}=5.8$.

\section{CONCLUSION}

Magnetically tunable metasurfaces for terahertz filtering that contain subwavelength resonators made of InAs, an anisotropic material, have been theoretically demonstrated. The transmission characteristics of the metasurface are strongly sensitive to the magnetostatic field varying from 0 to $1 \mathrm{~T}$. Combining effects of magnetostatic field strength and orientation, metasurfaces coupling, and dielectric substrate, one can realize various tuning and switching scenarios. The simulation results provide a good starting point for future experimental studies.

[1] S. Walia, C. M. Shah, P. Gutruf, et al., "Flexible metasurfaces and metamaterials: A review of materials and fabrication processes at microand nano-scales," Appl. Phys. Rev., vol. 2, art. no. 011303, 2015.

[2] R. J. Martin-Palma and A. Lakhtakia, Nanotechnology: A Crash Course, SPIE Press, Bellingham, WA, 2010.

[3] J. Han, A. Lakhtakia, and C.-W. Qiu, "Terahertz metamaterials with semiconductor split-ring resonators for magnetostatic tunability," Opt. Express, vol. 16, pp. 14390-14396, 2008.

[4] I. B. Vendik, O. G. Vendik, M. A. Odit, et al., "Tunable metamaterials for controlling THz radiation," IEEE Trans. Terahertz Sci. Technol., vol. 2, pp. 538-549, Sep. 2012.

[5] A. E. Serebriyannikov, A. Lakhtakia, and E. Ozbay, "Single and cascaded, magnetically controllable metasurfaces as terahertz filters," J. Opt. Soc. Am. B, vol. 33, at press, 2016. 